\title{
Termination of focal atrial tachycardia by adenosine triphosphate
}

\author{
MICHAEL S PERELMAN, DENNIS M KRIKLER \\ From the Department of Cardiovascular Disease, Royal Postgraduate Medical School, Hammersmith \\ Hospital, London
}

SUMMARY An atrial aneurysm gave rise to incessant atrial tachycardia in a 21 year old Seychellian soldier. This arrhythmia was suppressed by intravenous adenosine triphosphate and ajmaline. Resection of the aneurysm abolished the arrhythmia and re-established sinus rhythm.

Adenosine and its related compounds (such as adenosine triphosphate (ATP)) exert a potent effect on the atrioventricular node, slowing or even blocking conduction. This is the basis of their ability to terminate reciprocating atrioventricular tachycardias. Although in studies of myocytes isolated adenosine triphosphate inhibited focal atrial arrhythmias, no such effect has been reported in man. Indeed, it has been proposed that the response to adenosine triphosphate might clinically distinguish atrial arrhythmias from reciprocating atrioventricular tachycardias.

\section{Case report}

The patient, a fit 21 year old Seychellian soldier, experienced incessant rapid palpitation after exercise. Electrocardiograms consistently showed atrial tachycardia with considerable rate variation (120 to 280/minute). A 1:1 atrioventricular relation was usually retained (fig). The configuration of the $\mathbf{P}$ wave was abnormal and remained unchanged as the rate varied: it was broad $(160 \mathrm{~ms})$ with a mean frontal axis of $+60^{\circ}$, and inverted in V1 (fig). Most of the time he was well with only slight physical limitation, except during exercise when he became faint at more rapid rates. Verapamil, $\beta$-blockers, and disopyramide failed to suppress the arrhythmia, though verapamil sometimes produced partial atrioventricular block with a slower ventricular rate.

Requests for reprints to Dr Dennis M Krikler, Department of Cardiovascular Disease, Royal Postgraduate Medical School, Hammersmith Hospital, DuCane Road, London W12 0HS.
After a year he was referred to us. He was taking verapamil ( $160 \mathrm{mg}$ three times a day) and disopyramide $200 \mathrm{mg}$ (four times a day). On admission all medication was stopped and there was no change in rate or rhythm. Physical examination was completely normal apart from the persistent tachycardia. Routine blood tests, including those for thyroid function, were normal. Cross sectional echocardiography showed that all four valves were normal and that the chambers were of normal size, though there was mild left ventricular hypertrophy (posterior wall thickness $1.3 \mathrm{~cm}$ ).

At an electrophysiological study, during persistent tachycardia, mapping showed the earliest activity high and anteriorly in the right atrium. Carotid sinus massage (right and left) had no effect on the arrhythmia or conduction times. Both lying down from the sitting position and phase 1 of the Valsalva manoeuvre produced temporary high grade atrioventricular conduction block without changing the atrial cycle length.

After a bolus of intravenous adenosine triphosphate $(10 \mathrm{mg})$ the atrial rate slowed (AA interval lengthened from 400 to $500 \mathrm{~ms}$ ) and the tachycardia terminated with an associated shortening of the AH interval (fig). Sinus rhythm (normal $P$ wave configuration) took over for about 10 seconds when a $\mathbf{P}$ wave failed to be conducted (no subsequent $\mathbf{H}$ deflection). Ventricular escape was followed by an atrial extrasystole and reinitiation of the tachycardia.

Verapamil ( $10 \mathrm{mg}$ bolus) blocked AH conduction with no effect on the tachycardia, while ajmaline ( $50 \mathrm{mg}$ over 30 seconds) slowed and then terminated 


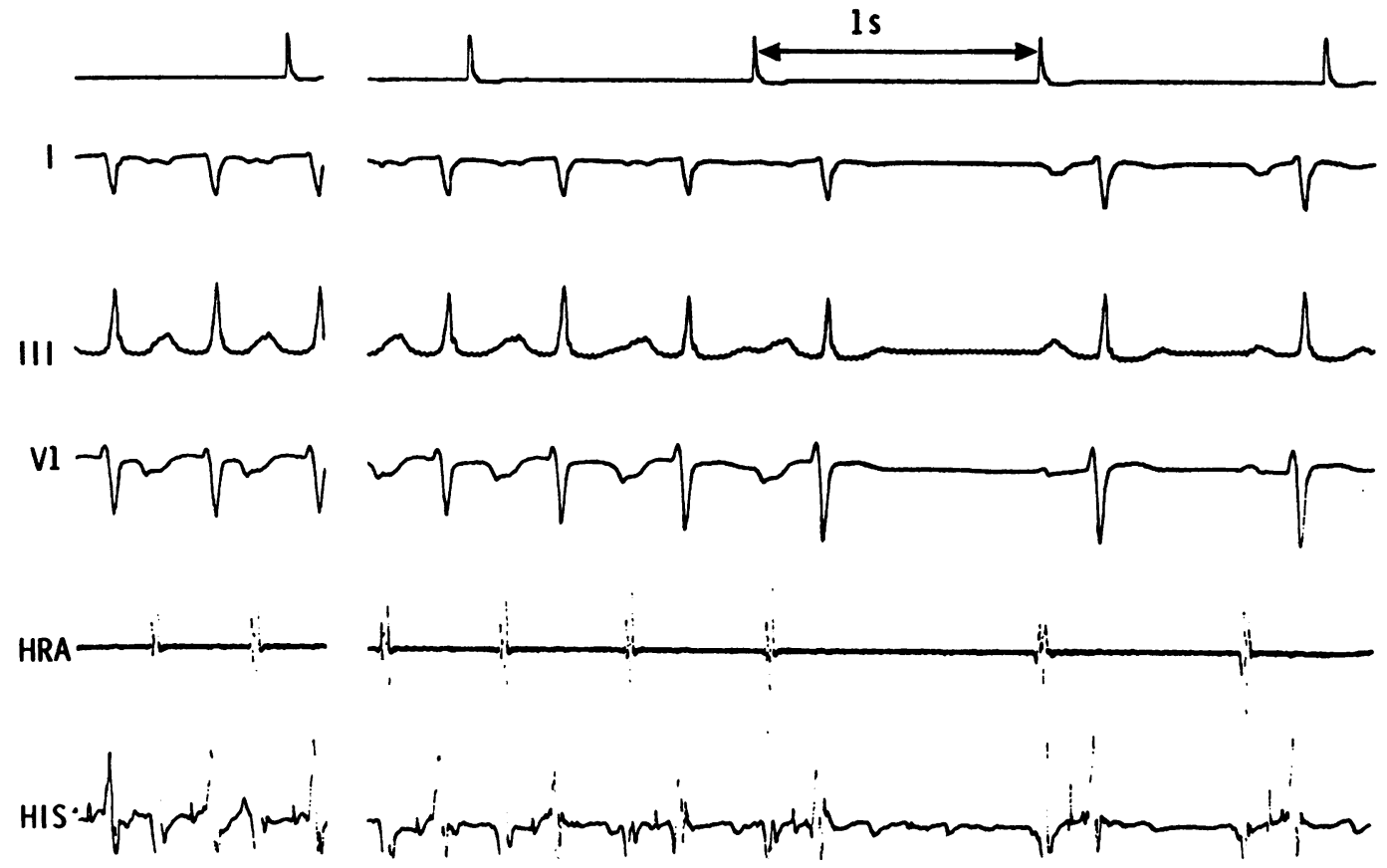

Figure Electrocardiogram showing atrial tachycardia; note termination after intravenous adenosine triphosphate. $I, I I I, V I$ : surface electrocardiograms; HRA: high right atrial electrogram; HIS: His electronram

the tachycardia; reinitiation occurred eight minutes later. Flecainide ( $2 \mathrm{mg} / \mathrm{kg}$ over eight minutes) did no more than temporarily slow the tachycardia.

We treated him with a combination of long-acting quinidine (Kinidin Durules, $250 \mathrm{mg}$ twice a day rising to $500 \mathrm{mg}$ twice a day) and verapamil $(40 \mathrm{mg}$ rising to $80 \mathrm{mg}$ four times a day). The atrial rhythm remained unchanged, but 2:1 atrioventricular block developed. Because the response was inadequate, he underwent operation ( $\mathrm{Mr} \mathrm{G}$ Bennett). A small $(1 \mathrm{~cm}$ diameter) atrial aneurysm was found and resected. This abolished the arrhythmia (follow up thirty months). Histological examination of the aneurysm (Dr E Olsen) showed normal atrial tissue with occasional inflammatory cells; there were no myocytes with histological features that suggested specialised function.

\section{Discussion}

Atrial tachycardias are by no means uncommon, though the need for surgical excision of the focus is unusual. ${ }^{1}$ Documented atrial aneurysms are rarely the cause of atrial tachycardias. Another unusual aspect of this case is the previously undocumented effect of adenosine triphosphate on an atrial arrhythmia, though, as indicated earlier, the effect of adenosine (and related compounds) on the atrioventricular node is well recognised and is the basis of their clinical use in the management of reciprocating atrioventricular tachycardias. ${ }^{2-4}$ Electrophysiological effects on atrial cells were demonstrated in the muscle bath: the action potential duration was shortened and the membrane was hyperpolarised ${ }^{56}$; upstroke velocity was unchanged ${ }^{5}$; intrinsic, automatic activity was depressed and spontaneous depolarisation inhibited ${ }^{67}$; while catecholamine induced triggered automaticity was suppressed. ${ }^{8}$ In man, however, no clinical or electrophysiological action on atrial arrhythmias has been demonstrated. Indeed, the lack of effect of adenosine and related compounds on atrial arrhythmias has been used to distinguish them from reciprocating atrioventricular tachycardias. ${ }^{49}$

Because experimental studies of adenosine triphosphate showed that it may suppress an atrial automatic focus or inhibit microreentry we cannot speculate either on the nature of the electrophysiological origin of the tachycardia in our case or on the exact mechanism by which adenosine triphosphate exerted 
530

its clinical effect. The case extends our knowledge of atrial tachycardias and suggests that in the future it would be worth giving adenosine triphosphate, or adenosine, to patients with atrial tachycardias in order to examine the response and perhaps to define further a subgroup of patients with atrial tachycardia in whom the mechanism may be unusual.

\section{References}

1 Olsson SB, Blomström P, Sabel K-G, William-Olsson G. Incessant ectopic atrial tachycardia: successful surgical treatment with regression of dilated cardiomyopathy picture. Am J Cardiol 1984;53:1465-6.

2 Berne RM, DiMarco JP, Belardinelli L. Dromotropic effects of adenosine and adenosine antagonists in the treatment of cardiac arrhythmias involving the atrioventricular node. Circulation 1984;69:1195-7.

3 Belhassen B, Pelleg A, Shoshani D, Geva B, Laniado S. Electrophysiologic effects of adenosine-5'-triphos-
Perelman, Krikler

phate on atrioventricular reentrant tachycardia. Circulation 1983;68:827-33.

4. DiMarco JP, Sellers TD, Lerman BB, Greenberg ML, Berne RM, Belardinelli L. Diagnostic and therapeutic use of adenosine in patients with supraventricular tachyarrhythmias. $\mathrm{J} \mathrm{Am}$ Coll Cardiol 1985;6:417-25.

5 Johnson EA, McKinnon MG. Effect of acetylcholine and adenosine on cardiac cellular potentials. Nature 1956;178:1174-5.

6 Belardinelli L, Isenberg G. Isolated atrial myocytes: adenosine and acetylcholine increase potassium conductance. Am J Physiol 1983;244:H734-7.

7 Hartzell HC. Adenosine receptors in frog sinus venosus. Slow inhibitory potentials induced by adenosine compounds and acetylcholine. J Physiol (Lond) 1979;293:23-49.

8 Belardinelli L, Isenberg G. Actions of adenosine and isoproterenol on isolated mammalian ventricular myocytes. Circ Res 1983;53:287-97.

9 Motté G, Waynberger M, Lebars A, Bouvrain Y. L'adenosine triphosphorique dans les tachycardies paroxystiques. Nouv Presse Med 1972;1:3057-61. 